\title{
Properties of and a New Technique for Fluorescent Detection of Influenza Virus Sialidase
}

\author{
Tadanobu Takahashi \\ Department of Biochemistry, School of Pharmaceutical Sciences, University of Shizuoka, \\ 52-1 Yada, Suruga-ku, Shizuoka-shi, Shizuoka 422-8526, Japan \\ FAX: +81-54-264-5722, E-mail: takahasi@u-shizuoka-ken.ac.jp
}

(Received on January 28, 2015, accepted on April 21, 2015)

Key Words: Influenza virus, neuraminidase, sialidase, pandemic, sialidase substrate

\begin{abstract}
Influenza A virus (IAV) has two envelope glycoproteins, hemagglutinin (HA) and neuraminidase (NA). HA recognizes sialic acids at the terminals of glycan chains on the host cell surface as virus receptors. NA shows sialidase activity, which cleaves sialic acids from the terminals of glycan chains. The viral sialidase activity is well-known to facilitate release of progeny virus from the host cell surface by preventing virus binding to sialic acid. Low-pH stability of viral sialidase activity is a unique property for pandemic IAV NAs distinct from most epidemic IAVs. It is thought that the low-pH stability of sialidase activity contributes to the spread of infection in a pandemic of a new subtype of IAV through enhancement of virus replication. Recently, a new sialidase substrate has been developed for histochemical fluorescent visualization of viral sialidase activity. This substrate can visualize living IAV-infected cells abundantly expressing viral NA by an easy protocol in a short time. The properties and functions of IAV sialidase activity, mainly in terms of low-pH stability, and a new tool for detection of IAV sialidase activity are described in this review.
\end{abstract}

\section{A. Introduction}

Glycan chains and glyco-metabolic enzymes play important roles in virus infections. For example, sialic acids at the terminals of glycan chains are receptors for attachment of influenza virus to the host cell surface, meaning that glycan chains have functions as viral receptors. Influenza viruses are classified into A, B, and $\mathrm{C}$ types by viral internal antigens. Influenza A virus (IAV) is a respiratory pathogen that can infect various hosts including humans, birds, pigs, and horses. IAV has two envelope glycoproteins, hemagglutinin (HA) and neuraminidase (NA). HA recognizes sialic acids on the host cell surface. This binding initiates cell entry of viral particles via an endocytic pathway. Acidification in endosomes/ lysosomes induces the fusion process between the viral membrane and cellular membrane through exposure of the fusion peptide in HA resulting from conformation change of HA. Viral genomes enter the nucleus, where viral genomes replicate by viral RNA polymerase. The replicated viral genome forms viral ribonucleoprotein complexes (vRNP) together with three viral RNA polymerase subunits and viral nucleoprotein (NP), and the complexes are exported to the cytosol across the nuclear membrane (1). Nascent HA and NA are expressed on the surfaces of infected cells. Components of progeny viruses including vRNP are also transported to the cell surface. Progeny virus particles are budded from the cell surface membrane together with the host lipid bilayer and viral components. NA has high sialidase activity, which cleaves terminal sialic acids from glycan chains. NA is known to facilitate the release of progeny viruses from the host cell surface by preventing binding of the viruses to sialic acids $(2,3)$. NA also suppresses self- aggregation between progeny viruses by removal of sialic acids on HA and NA of the progeny viruses (4). Inhibition of sialidase activity results in a significant reduction of IAV replication. Currently, viral sialidase inhibitors are clinically used as influenza virus-specific medicines such as zanamivir (5).

Antigenicities of the viral envelope glycoproteins have so far been classified into eighteen subtypes for HA and eleven subtypes for NA. N10 and N11 subtypes of NA show no sialidase activity (6-8). Viral genomes of IAV are eight-segmented negativestranded RNA. When more than two viruses infect the same cell, their viral genomes can be replaced among the viruses within the infected cell. Viral genome replacement of HA and/or NA genes confers drastic change of the viral surface antigenicities, resulting in potential occurrence of a pandemic as a new subtype of IAV. Since the 20th century, there have been four pandemics: Spanish Flu (H1N1) in 1918, Asian Flu (H2N2) in 1957, Hong Kong Flu (H3N2) in 1968, and pandemic (H1N1) 2009 in 2009. H1N1 and H3N2 are currently the main subtypes in human IAVs. H2N2 IAV also became epidemic among humans in the past.

\section{B. Structure of IAV NA}

IAV NAs are phylogenetically classified into group 1 (N1, N4, N5, and N8) and group 2 (N2, N3, N6, N7, and N9). IAV NA, a type II transmembrane glycoprotein of approximately 470 amino acids, possesses four partial domains, a globular domain (approximately 390 amino acids), a stalk region, a cytoplasmic tail (CT, 6 amino acids of residue numbers 1-6), and a transmembrane domain (TMD, 29 amino acids of residue numbers 7-35). The func- 
tion of the short CT sequence that is highly conserved among IAV NAs remains unclear. The hydrophobic TMD is an apical sorting signal in polarized epithelial cells and anchor domain for membrane binding through interaction with lipid rafts $(9,10)$. Until 2010, amino acid sequences of the NA stalk regions are classified into six patterns: $15,16,19,20$, and 22-amino-acid deleted stalks, and a full length stalk (44 amino acids of residue numbers 36-79 based on NA numbering of A/goose/Guangdong/1/1996 H5N1 strain) $(11,12)$. For the stalk regions of H5N1 highly pathogenic avian IAV (HPAI) NAs, all H5N1 isolates in 2007 possessed a 20-amino-acid deletion. The short-length stalk increases virulence and pathogenicity of IAV in mice $(12,13)$. The globular domain is a mushroom-shaped structure including the enzymatic active site that is highly conserved among all subtypes of IAVs and influenza $\mathrm{B}$ viruses. A homodimer is formed by an intermolecular disulfide bond in the stalk $(14,15)$. Native NA on the viral surface exists as a homotetramer (identical monomers with molecular weights of approximately 50,000-60,000), which is an essential structure for enzymatic activity (16). The highly conserved amino acid residues in the globular domain of N2 NA, including the active site residues, are shown in detail in Table 5 of a report by Varghese et al. in 1991 (3). It is thought that these residues are essential for the function of IAV NA.

In 2009 and 2010, H17N10 and H18N11 IAVs were isolated from a little yellow-shouldered bat and a flat-faced fruit bat, respectively. N10 and N11 NA genes are highly divergent from all NAs from N1 to N9. N10 and N11 NAs are called NA-like proteins because of their lack of sialidase activity (6-8).

\section{Properties of IAV Sialidase Activity}

\section{C-1. Absolute Sialidase Activity}

Absolute sialidase activities of avian IAV NAs and 1957 pandemic NA (directly derived from avian IAV NA) are more than 10-times higher than those of general epidemic human IAV NAs. Six amino acid alterations in N2 NA of A/England/12/1962 (H2N2) converted the low sialidase activity to high sialidase activity like that of the 1957 pandemic A/Singapore/1/1957 (H2N2). These amino acid residues were not necessarily located near the active site. Avian IAV usually infects and replicates in waterfowl intestines through oral inoculation. High absolute activity of avian NA seems to be required for replication ability of avian IAV in the duck intestine after oral inoculation (17). On the other hand, transition to low absolute activity that appeared in human epidemic IAV may be required for host adaption of IAV to humans. In addition, deletion in the stalk region of HPAI A/Hong Kong/486/1997 (H5N1) NA conferred higher absolute sialidase activity than that of the parent long-stalk NA (13). On the other hand, deletion in the stalk region of avian IAV A/Mallard/Marquenterre/Z237/1983 (H1N1) NA resulted in absolute sialidase activity similar to that of the parent long-stalk NA (18). Change in absolute sialidase activity by stalk-length of NA is likely dependent on virus strains. Also, the absence of $N$-linked glycosylation at amino acid position 130 (position 146 by N2 numbering) of A/WSN/1933 (H1N1) NA increased the absolute sialidase activity. The $N$-linked glycosylation at this site close to the active site probably prevents access of the substrate to the active site (19).

\section{C-2. Effect of Cations}

Divalent cations affect the sialidase activity of NAs from certain sources such as bacteria. There are one to three calcium ion binding sites on the monomers of IAV NAs. Calcium ions increase the sialidase activity of IAV and influenza B virus NAs. The presence of calcium ions also makes NAs resistant to thermal inactivation $(20,21)$. Calcium binding sites with low affinity are located on the tetramer fourfold symmetry axis neighboring the subunit interfaces, and calcium binding sites with high affinity are close to the active site on each monomer. Therefore, binding of calcium ions on NA is thought to be involved in structure stabilization of the active site in each monomer and the entire tetramer. Sensitivities of sialidase activity to calcium ions are different among NA subtypes. Magnesium ions behave similarly to calcium ions. Zink ions have a slightly inhibitory effect on the sialidase activity of A/ Tokyo/3/1967 (H2N2) but have no effect on N1 NA. Other monovalent cations, such as potassium ions, sodium ions and tetrabutyl ammonium ions, have little effect on N2 NA activity $(22,23)$. Calcium ions are also involved in maintenance of immunogenicity of N1 and N2 NAs, suggesting that calcium ions are important for host immunization against NA antigen $(22,24)$.

\section{C-3. Substrate Specificity for Sialyl Linkages}

IAV recognizes sialyl linkages of the terminal sialic acid $\mathrm{N}$-acetylneuraminic acid (Neu5Ac) to galactose (Gal), Neu5Ac $\alpha 2,3 \mathrm{Gal}$ and Neu5Ac $\alpha 2,6 \mathrm{Gal}$. Neu5Ac $\alpha 2,3 \mathrm{Gal}$ is abundantly expressed in the chicken trachea and duck intestine. Neu5Ac $\alpha 2,6 \mathrm{Gal}$ is rich in the upper respiratory tract of humans. The pig trachea contains both Neu5Ac $\alpha 2,3 \mathrm{Gal}$ and Neu5Ac $\alpha 2,6 \mathrm{Gal}$. Avian IAV and human IAV preferentially bind to Neu5Ac $\alpha 2,3 \mathrm{Gal}$ and Neu5Ac $\alpha 2,6 \mathrm{Gal}$, respectively. Swine IAV binds to both receptors. This binding specificity is believed to determine host specificity (1). Sialidase activity of IAV NA can also discriminate sialyl linkages of Neu5Ac $\alpha 2,3 \mathrm{Gal}$ and Neu5Ac $\alpha 2,6 \mathrm{Gal}$. However, IAV sialidase activity basically has a more preferential specificity for Neu5Ac $\alpha 2,3 \mathrm{Gal}$ than for Neu5Ac $\alpha 2,6 \mathrm{Gal}$ of various substrates such as sialyllactose (Neu5Ac $\alpha 2,3 \mathrm{Gal} \beta 1,4 \mathrm{Glc}$ or Neu $5 \mathrm{Ac} \alpha 2,6 \mathrm{Gal}$ $\beta 1,4 \mathrm{Glc}$ ) or sialyllactosamine (Neu5Ac $\alpha 2,3 \mathrm{Gal} \beta 1,4 \mathrm{GlcNAc}$ or Neu5Ac $\alpha 2,6 \mathrm{Gal} \beta 1,4 \mathrm{GlcNAc})$. NAs of avian and 1957 pandemic 
human IAV are resistance to hydrolysis of Neu5Ac $\alpha 2,6 \mathrm{Gal}$ (25, 26). Human IAV N2 NAs, which first appeared in 1957, gradually increased the substrate specificity for Neu5Ac $\alpha 2,6 \mathrm{Gal}$ until human IAV N2 NAs in 1987, although the recognition was lower than that for Neu5Ac $\alpha 2,3 \mathrm{Gal}$. For N1 NA, the 1918 pandemic human IAV showed higher substrate specificity for Neu5Ac $\alpha 2,6 \mathrm{Gal}$ than that of other avian IAVs (27). For 1957 pandemic A/Singapore/1/1957 (H2N2) NA, increased specificity for Neu5Ac $22,6 \mathrm{Gal}$ was conferred by an single amino acid alteration from Ile to Val at amino acid position 275, which is close to the glycerol group of Neu5Ac bound within the active site $(25,26,28,29)$. Some swine IAV N2 NAs also have specificity for Neu5Ac $\alpha 2,6$ Gal like human IAVs $(26,30)$. For the release process of progeny virus, high sialidase activity should be needed for preventing strong receptor binding. However, the IAV receptor binding specificity is not necessarily in accordance with the IAV NA substrate specificity for Neu5Ac $\alpha 2,3 \mathrm{Gal}$ and Neu5Ac $\alpha 2,6 \mathrm{Gal}$, especially in human IAV. It is suspected that the high sialidase activity for Neu5Ac $\alpha 2,3 \mathrm{Gal}$ plays an important role in the IAV life cycle, probably even in processes other than the release process of progeny IAV.

\section{C-4. Substrate Specificity for Sialic Acid Species}

Sialic acids recognized by IAV are mainly divided into Neu5Ac and $N$-glycolylneuraminic acid (Neu5Gc) by the presence or absence of a hydroxyl group at the $\mathrm{N}$-acetyl group added to position 5 of the sialic acid moiety. Humans cannot synthesize Neu5Gc because of a genetic deficiency of the Neu5Gcsynthesizing enzyme, cytidine-5'-monophospho-Neu5Ac hydroxylase. Neu5Gc has been believed to be a receptor of human $(31,32)$, swine (33), duck (34), and equine IAVs (35), because of Neu5Gc binding activity of IAVs and Neu5Gc expression in IAV replication tissue. However, a recent studies has shown that Neu5Gc expressed genetically or metabolically on human and pig epithelial cells had an inhibitory effect on infection of IAV with Neu5Gc binding ability (36). It has been suggested that Neu5Gc is a decoy receptor but not a functional receptor of IAV with Neu5Gc binding ability (37). N2 NAs of human, swine, and avian IAVs show lower specificity for Neu5Gc $\alpha 2,3 \mathrm{Gal}$ than for Neu5Ac $\alpha 2,3 \mathrm{Gal}$, using the ganglioside GM3 containing Neu5Gc (Neu5Gc $\alpha 2,3 \mathrm{Gal} \beta 1,4 \mathrm{Glc}$ $\beta 1 \mathrm{Cer}$ ) or $\mathrm{Neu} 5 \mathrm{Ac}(\mathrm{Neu} 5 \mathrm{Ac} \alpha 2,3 \mathrm{Gal} \beta 1,4 \mathrm{Glc} \beta 1 \mathrm{Cer})$ as a sialidase substrate. For substrate specificity of N2 NA for Neu5Gc $\alpha 2,3 \mathrm{Gal}$, avian IAV is lower than those of swine IAV. 1968 pandemic H3N2 IAV shows higher substrate specificity than early human H2N2 IAVs between 1957 and 1962 and human epidemic H3N2 IAVs after 1972. For A/England/12/1962 (H2N2) NA, increased specificity for Neu $5 \mathrm{Gc} \alpha 2,3 \mathrm{Gal}$ was conferred by an single amino acid alteration from Gln to Lys at amino acid position 431, which is far above the active site and is located toward a carboxyl group of Neu5Gc bound within the active site (26). The relationship between binding specificity and sialidase specificity of IAV for Neu5Gc is not clear. Since Neu5Gc expression on the host cells has an inhibitory effect on IAV infection, sialidase activity cleaving Neu5Gc may have an advantage effect on the infection process.

\section{C-5. Other Substrate Specificities}

IAV NA discriminates the internal structure and length of sugar chains. 1968 pandemic A/Aichi/2/1968 (H3N2) NA recognizes the lacto-series sugar chain type I (Neu5Ac $\alpha 2,3 \mathrm{Gal}$ $\beta 1,3 \mathrm{GlcNAc} \beta 1,3 \mathrm{Gal} \beta 1,4 \mathrm{Glc}$ ) and type II (Neu5Ac $\alpha 2,3 \mathrm{Gal}$ $\beta 1,4 \mathrm{GlcNAc} \beta 1,3 \mathrm{Gal} \beta 1,4 \mathrm{Glc})$ more specially than the ganglioseries sugar chain (Neu5Ac $\alpha 2,3 \mathrm{Gal} \beta 1,3 \mathrm{GalNAc} \beta 1,3 \mathrm{Gal} \beta 1,4 \mathrm{Glc}$ ) and specifically hydrolyze the terminal Neu5Ac $\alpha 2,3 \mathrm{Gal}$ in longchain gangliosides. Sialidase activities of A/Puerto Rico/8/1934 (H1N1) and A/Aichi/2/1968 (H3N2) cannot hydrolyze Neu5Ac $\alpha 2,3 \mathrm{Gal}$ or Neu5Ac $\alpha 2,8 \mathrm{Neu} 5 \mathrm{Ac} \alpha 2,3 \mathrm{Gal}$ linked to the inner galactose residue of ganglioside GM1a [Gal $\beta 1,3$ GalNAc $\beta 1,4$ $(\mathrm{Neu} 5 \mathrm{Ac} \alpha 2,3) \mathrm{Gal} \beta 1,4 \mathrm{Glc} \beta 1 \mathrm{Cer}]$ or GD1b $[\mathrm{Gal} \beta 1,3 \mathrm{GalNAc} \beta 1,4$ (Neu5Ac $\alpha 2,8 \mathrm{Neu} 5 \mathrm{Ac} \alpha 2,3$ ) Gal $\beta 1,4 \mathrm{Glc} \beta 1 \mathrm{Cer}]$. Neu5Ac $\alpha 2,3 \mathrm{Gal}$ linked to the inner galactose of the shorter chain of GM2 [GalNAc $\beta 1,4$ (Neu5Ac $\alpha 2,3$ ) Gal $\beta 1,4 \mathrm{Glc} \beta 1 \mathrm{Cer}]$ can be hydrolyzed by $\mathrm{A} /$ Aichi/2/1968 (H3N2), though at a relatively low rate (29). GT1b [Neu5Ac $\alpha 2,3 \mathrm{Gal} \beta 1,3 \mathrm{GalNAc} \beta 1,4$ (Neu5Ac $\alpha 2,8 \mathrm{Neu} 5 \mathrm{Ac} \alpha 2,3$ ) $\mathrm{Gal} \beta 1,4 \mathrm{Glc} \beta 1 \mathrm{Cer}]$ and GD1a [Neu $5 \mathrm{Ac} \alpha 2,3 \mathrm{Gal} \beta 1,3 \mathrm{GalNAc}$ $\beta 1,4$ (Neu5Ac $\alpha 2,3) \mathrm{Gal} \beta 1,4 \mathrm{Glc} \beta 1 \mathrm{Cer}]$ are primarily converted to GD1b and GM1a by sialidase activity of A/Leningrad/385/1980 (H3N2), respectively (38). IAV NA has also been reported to discriminate the ceramide moiety of sialyl glycolipids. Sialidase activity of A/Aichi/2/1968 (H3N2) hydrolyzes GM3 with a hydroxyl group at the $\beta$ position in the fatty acid side chain more rapidly than that with a hydroxyl group at the $\alpha$ position (29). There are $N$ linked and $O$-linked glycans on glycoproteins. Sialic acids of $\alpha-1$ acid glycoprotein and fetuin-bearing $N$-linked glycan are sensitive to hydrolysis of IAV NA, whereas those of mucin-bearing $O$-linked glycan are more resistant to hydrolysis of IAV NA $(25,27)$. The resistance of $O$-linked glycan to IAV NA may be helpful to host defense at the mucosa to trap IAV through binding to sialic acids on mucin.

\section{The Low-pH Stability of IAV Sialidase Activity D-1. Various Low-pH Stabilities of IAV Sialidase Ac- tivity}

IAV NAs show different low-pH stabilities in the sialidase activity of each virus strain. Low-pH stability of IAV NA means the property to maintain sialidase activity under an acidic condition between $\mathrm{pH} 4.0$ and 5.0. The low-pH stability does not affect optimal $\mathrm{pH}$ ( $\mathrm{pH}$ of approximately 6.0) of IAV sialidase activity (39). 
For human IAV, almost all epidemic IAVs quickly and irreversibly lose sialidase activity by incubation at $37^{\circ} \mathrm{C}$ within $10 \mathrm{~min}$ in the acidic condition of $\mathrm{pH} 4.0$ (even $\mathrm{pH} 5.0$ for some virus strains). On the other hand, all of the pandemic IAVs in 1918, 1957, 1968, and 2009 have low-pH-stable NA that maintains sialidase activity even with incubation at $37^{\circ} \mathrm{C}$ for $10 \mathrm{~min}$ at $\mathrm{pH} 4.0$ (39-41). 1918 pandemic IAV NA has Leu or Phe at amino acid position 256 (based on 1918 IAV NA numbering) (42). This amino acid difference has no effect on the low-pH stability (40). Swine IAV shows no lowpH stability in sialidase activity as does human epidemic IAV (39). Avian IAV, except most H5N1 HPAIs, has strong low-pH-stable NA that does not decrease sialidase activity even with incubation at $37^{\circ} \mathrm{C}$ for $1 \mathrm{~h}$ at $\mathrm{pH} 4.0$ (Fig. 1). Strangely, almost all H5N1 HPAIs, regardless of isolated hosts including humans, chickens and water birds, show no low-pH stability in sialidase activity like human epidemic IAV (43). Some H5N1 HPAIs have low-pH-stable NA that moderately decreases sialidase activity at $\mathrm{pH} 4.0$ in an incubation time-dependent manner for more than 10 min. Such a lowpH-stable NA also similarly appeared in pandemic IAV (40, 43). Human epidemic H1N1 IAV after the 1918 pandemic temporarily disappeared in the 1950s. In 1977, re-emergence of human H1N1 IAV had often been called Russian Flu as one of pandemic. Generally, a new subtype IAV in a pandemic readily infects many people

\section{Avian IAV}
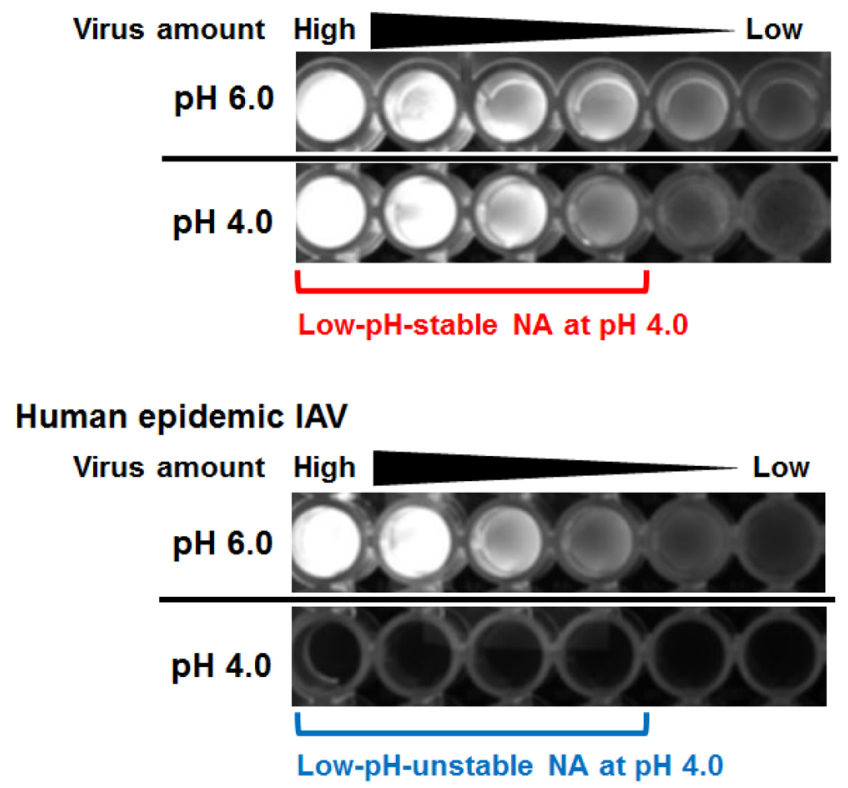

Fig. 1. Low-pH stability of IAV sialidase activity. Two-fold serial dilutions of avian IAV A/duck/Hong Kong/24/5/1976 (H3N2) (upper) and human epidemic IAV A/Memphis/1/1971 (H3N2) (lower) were incubated at $\mathrm{pH} 4.0$ or 6.0 ( $\mathrm{pH} 6.0$ being near to optimal $\mathrm{pH})$. Sialidase activity was fluorescently visualized under UV irradiation by reaction with $4 \mathrm{MU}-\mathrm{Neu} 5 \mathrm{Ac}$. Avian IAV sialidase activity shows low-pH stability at $\mathrm{pH} 4.0$. without immunization against the subtype and spreads worldwide. However, 1977 H1N1 IAV was genetically identical to human epidemic H1N1 IAV that disappeared in the 1950s. Many people more than 25 years of age had already acquired immunization of 1918-1950s H1N1 IAV. Infection of 1977 H1N1 IAV was limited to young people less than 25 years old $(44,45)$. In addition, 1977 H1N1 IAV does not have the low-pH-stable NA that appeared in pandemic IAVs (40). In some aspects including the low stability of sialdiase activity, 1977 H1N1 IAV is apparently different from other pandemic IAVs, thus suggesting that 1977 H1N1 IAV is close to epidemic IAV rather than pandemic IAV. H1N1 IAV (called A USSR type) derived from 1977 IAV had continued to be epidemic until the occurrence of new H1N1 pandemic in 2009. For the 2009 pandemic, almost all of the new H1N1 IAVs isolated in the United States after late April in 2009 had low-pH-stable NA. On the other hand, almost all of the new H1N1 IAVs in March and before early April in 2009 did not have low-pH-stable NA (41).

\section{D-2. Strong Low-pH Stability of Avian IAV Sialidase Activity}

Duck IAV passes through an acidic condition in the gizzard $(\mathrm{pH} 3.6-4.5)$ after oral inoculation to a duck and replicates in the duck intestine with no symptoms of infection (46). Human IAV cannot infect ducks. Conversely, duck IAV cannot infect humans. Thus, the IAV host is strictly restricted (47). Two IAV strains with the low-pH-stable NA gene of human IAV A/Singapore/1/1957 (H2N2) or A/Ann Arbor/6/1960 (H2N2) and seven other genes of duck IAV A/duck/Hong Kong/278/1978 (H2N9) can replicate in the duck intestine through oral inoculation, but an IAV strain with the NA gene of human IAV A/Korea/426/1968 (H2N2) and seven other genes of duck IAV A/duck/Hong Kong/278/1978 (H2N9) cannot (17). The NA of A/Korea/426/1968 (H2N2) is phylogenetically very close to the low-pH-unstable NA of human IAV A/ Taiwan/1/1964 (H2N2) (48). It is predicted that A/Korea/426/1968 (H2N2) NA is low-pH-unstable like human epidemic IAV and that the low-pH-stable NA is required for replication of avian IAV in the duck intestine after passing through the duck digestive tract. Maintenance of sialidase activity after passing through the gizzard may prevent the arrest of IAV by sialic acids on digestive mucin until the IAV reaches the duck intestine. Although the reason for no low-pH stability in almost all H5N1 HPAI NAs is unknown, it is thought that the strong low-pH stability of avian IAV NA plays a significant role in host restriction of the duck.

D-3. Amino Acid Residues Responsible for Low-pH Stability of IAV Sialidase Activity

Amino acid residues responsible for the low-pH stability of sialidase activity have been identified in some NAs. In the low-pHstable NA of 1918 pandemic A/Brevig Mission/1/1918 (H1N1), 
two amino acid alterations of Gln to Arg at position 430 and Gly to Asn at position 454 and insertion of Thr at position 435 resulted in loss of the low-pH stability of sialidase activity (based on A/ Brevig Mission/1/1918 NA numbering). Conversely, in the lowpH-unstable NA of A/USSR/92/1977 (H1N1), two amino acid alterations of Arg to Gln at position 430 and Asn to Gly at position 455 and a deletion of $\mathrm{Thr}$ at position 435 resulted in the acquisition of low-pH stability to a level similar to that in 1918 pandemic IAV (based on A/USSR/92/1977 NA numbering) (40). The positions of 430 and 435 are near the active site, the calcium ion-binding site, and the subunit interfaces between NA monomers. The position of 454 (or 455) is one of the amino acid residues constituting the subunit interfaces (Fig. 2) (49).

In the low-pH-stable NA of 1968 pandemic A/Hong Kong/1/1968 (H3N2), two amino acid alterations of Arg to Lys at position 344 and Phe to Leu at position 466 resulted in loss of the low-pH stability in sialidase activity. In the low-pH-unstable NA of human epidemic A/Texas/1968 (H2N2), a single amino acid alteration of Leu to Phe at position 466 is sufficient for acquisition of low-pH stability to a level similar to that in A/Hong Kong/1/1968 (H3N2) (based on N2 NA numbering of human IAV) (50). The position of 344 is near the active site and the calcium ion-binding site $(3,23,51)$. The position of 466 is near the subunit interfaces between NA monomers (Fig. 2) (52-54).

2009 pandemic IAVs isolated from March to May in 2009 included two types of IAVs with low-pH-stable NA and low$\mathrm{pH}$-unstable NA. There are only two amino acid differences between the low-pH-unstable NA of 2009 pandemic A/California/04/2009 (H1N1) and the low-pH-stable NA of 2009 pandemic A/Norway/3858/2009 (H1N1). In the low-pH-unstable NA of A/ California/04/2009 (H1N1), two amino acid alterations of Val to Ile at position 106 and Asn to Asp at position 248 resulted in the acquisition of low-pH stability to a level similar to that in $\mathrm{A} /$ Norway/3858/2009 (H1N1) (based on N1 NA numbering of 2009 pandemic IAV) (41). The position of 106 is the inner residue near the calcium ion-binding site and the subunit interfaces between NA monomers. The position of 248 is near the active site and the calcium ion-binding site (Fig. 2) (55).

Some H5N1 HPAIs have low-pH-stable NA like pandemic IAV NA, although almost all H5N1 HPAI NAs show no low-pH stability. In the low-pH-stable NA of A/duck/Guangdong/1/2001 (H5N1), two amino acid alterations of Tyr to His at position 155 and Asn to Lys at position 341 resulted in loss of the low-pH stability in sialidase activity. In the low-pH-unstable NA of A/ Hong Kong/213/2003 (H5N1), the reverse alterations resulted in acquisition of low-pH stability to a level similar to that in $\mathrm{A} /$ duck/ Guangdong/1/2001 (H5N1) (based on N1 NA numbering of A/
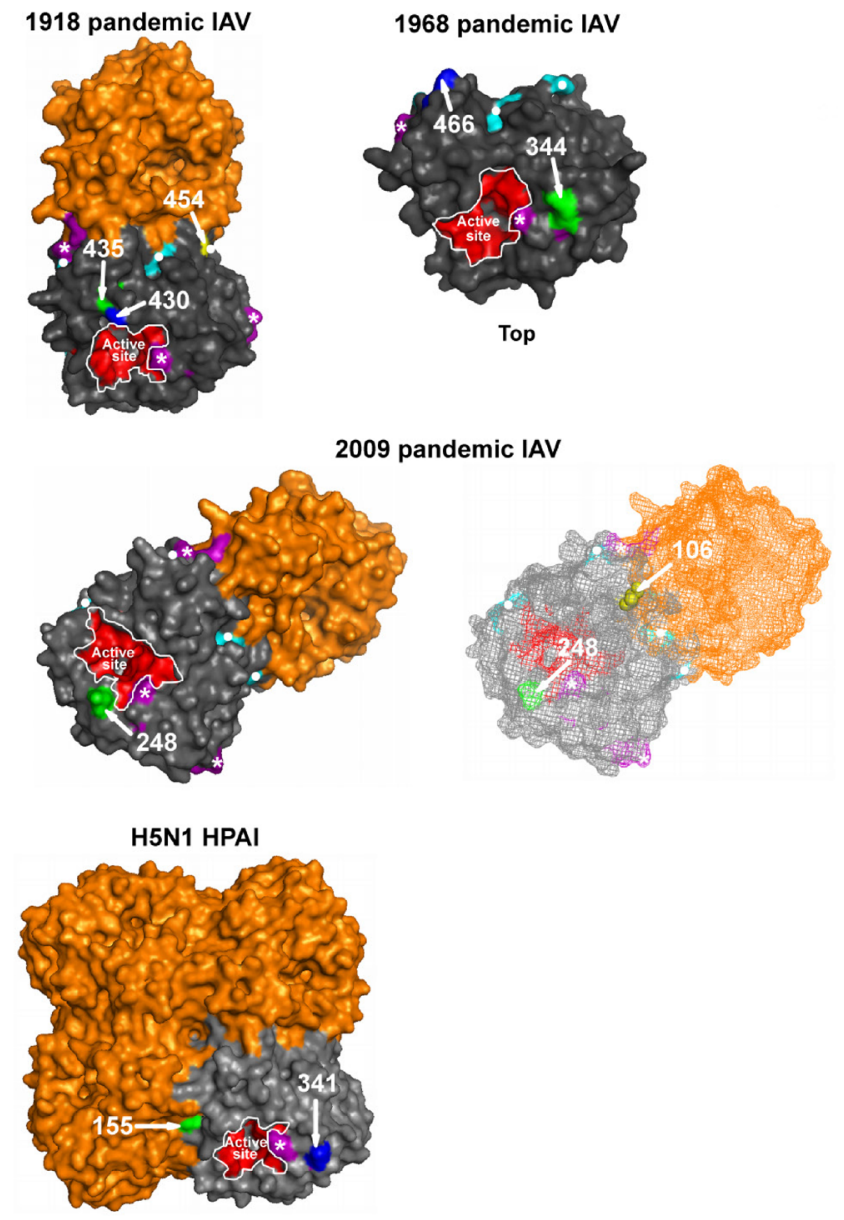

Fig. 2. Amino acid positions responsible for the low-pH stabilities of IAV sialidase activity. An asterisk and white circle show amino acid positions of the calcium ion-binding site and the subunit interfaces exposed on the surface of the NA three-dimensional structure, respectively. The surface model of the NA structure was generated by using the Pymol Molecular Graphics System Ver. 1.1r1 (DeLano Scientific LLC). Numbers indicate amino acid position numbers responsible for the low-pH stability of sialidase activity (based on each NA numbering). For 1918 pandemic IAV NA, the homodimer structure of A/Brevig Mission/1/1918 (H1N1) NA (PDB ID, 3BEQ) was used. For 1968 pandemic IAV NA, the monomeric structure of A/Tokyo/3/67 (H2N2) NA (PDB ID, 2BAT) close to NAs of 1968 pandemic NA at the amino acid sequence level was used. For 2009 pandemic NA, the homodimer structure of A/California/04/2009 (H1N1) NA (PDB ID, 3NSS) was used. The structure on the right side is meshed to view the sphere-modeled residue at position 106 inside the NA dimer. For H5N1 HPAI NA, the homotetramer structure of A/Viet Nam/1203/2004 (H5N1) NA (PDB ID, 2HTY) was used.

duck/Guangdong/1/2001 and A/Hong Kong/213/2003) (43). The position of 155 is near the subunit interfaces between NA monomers. The position of 341 is near the active site and the calcium ion-binding site (Fig. 2) (56).

One to three amino acid alterations in NA confer loss or acquisition of low-pH stability in IAV sialidase activity. Positions of these amino acids are near the active site, the calcium ion-binding 
site, and/or the subunit interfaces. It is thought that amino acid alterations affecting the three-dimensional structure of the active site and the homotetramer in IAV NA dramatically change the low$\mathrm{pH}$ stability. Since dramatic change in the low-pH stability can be invoked by one to three amino acid alterations only in NA, such a change in low-pH stability may occur easily in natural IAV.

\section{D-4. Function of the Low-pH Stability of IAV Siali- dase Activity}

Viral replication ability was investigated using IAVs with loss or acquisition of low-pH stability by genetic amino acid alterations. A/WSN/1933 with the low-pH-unstable NA gene of A/USSR/92/1977 (H1N1) shows lower replication ability and smaller plaque than those of the corresponding IAV with the lowpH-stable NA gene modified by two alterations of Arg430Gln and Asn455Gly and a deletion of Thr435 in Madin-Darby canine kidney (MDCK) cells, which are often used for culture of and experiments on IAV (40). Large size of the viral plaque means high replication ability and/or high cytotoxic ability of IAV. In MDCK cells, A/WSN/1933 with the low-pH-stable NA gene of A/ Hong Kong/1/1968 (H3N2) shows higher replication ability and larger plaque than those of the corresponding IAV with the low$\mathrm{pH}$-unstable NA gene modified by two amino acid alterations of Arg344Lys and Phe466Leu. Conversely, in MDCK cells, A/ WSN/1933 with the low-pH-unstable NA gene of A/Texas/1968 (H2N2) shows lower replication ability and smaller plaque than those of the corresponding IAV with the low-pH-stable NA gene modified by a single amino acid alteration of Leu466Phe. Two
IAVs with these low-pH-stable N2 NAs efficiently replicated in the mouse lung by intranasal inoculation. Replication of other IAVs with these low-pH-unstable N2 NAs was not detected in the mouse lung (57). In MDCK cells and human lung adenocarcinoma (calu3) cells, A/California/04/2009 (H1N1) with low-pH-unstable NA shows lower replication ability and smaller plaque than those of the corresponding IAV with low-pH-stable NA modified by two amino acid alterations of Val106Ile and Asn248Asp (41).

Taken together, the results show that IAV with low-pH-stable NA has much higher replication ability and larger plaque than those of IAV with low-pH-unstable NA (Fig. 3). IAVs with the low-pH-stable NA of A/Hong Kong/1/1968 (H3N2) or the modified low-pH-stable NA (Leu466Phe) of A/Texas/1968 (H2N2) maintain sialidase activity under an endocytic pathway (cell entry), known as an intracellular acidic compartment near or below $\mathrm{pH} 5.0$ (58-60). On the other hand, the corresponding IAVs with the lowpH-unstable NA of A/Texas/1968 (H2N2) or the modified low-pHunstable NA (Arg344Lys and Phe466Leu) of A/Hong Kong/1/1968 (H3N2) lose sialidase activity immediately after initiation of endocytosis. Inhibition of viral sialidase activity under an endocytic pathway by IAV and an influenza B virus NA-specific inhibitor, zanamivir, decreases progeny IAV production in the infected cells (57). It is thought that viral sialidase activity under an endocytic pathway plays an important role in IAV production and replication. Sialidase activity of IAV is well-known to be functional for facilitation of the release of progeny virus budded on the cell surface and for prevention of self-aggregation among progeny viruses, at

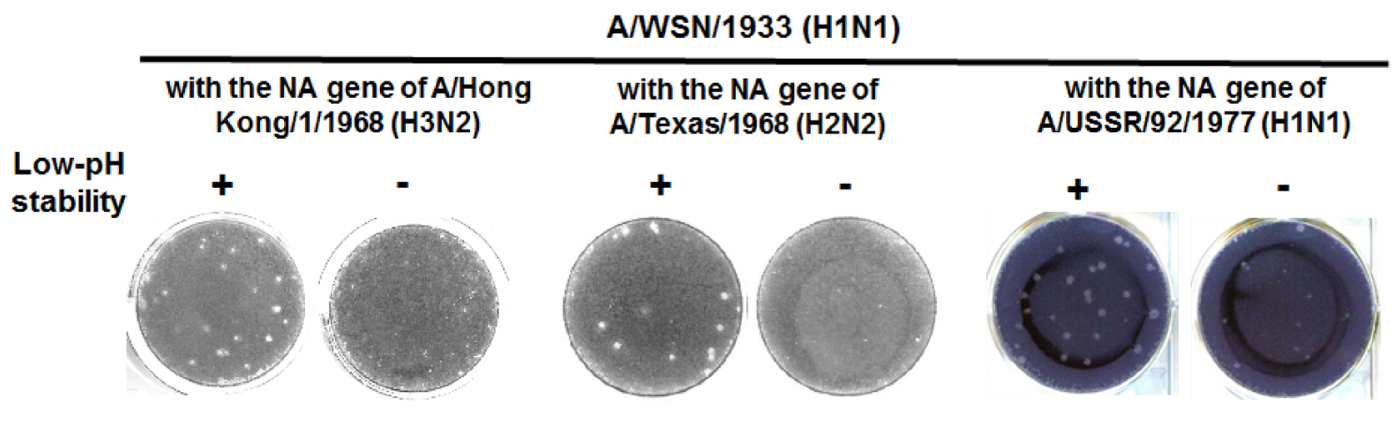

A/California/04/2009 (H1N1)

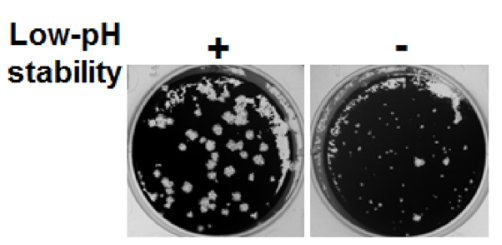

Fig. 3. Plaque images of IAVs with low-pH-stable NA or low-pH-unstable NA. MDCK cells were infected with each IAV. Virus-infected cells were cultured in $0.5-0.8 \%$ agarose-containing medium in the presence of acetylated trypsin for 2 or 3 days. Cells were fixed and stained with Crystal Violet or amide black. Dead infected cells are visualized as a plaque because it is not stained by dropout of dead cells. Large plaques of IAVs with low-pH-stable NA mean high replication ability of the IAVs. 
the late stage of the IAV lifecycle. On the other hand, some studies, including a study on the low-pH stability of IAV NA, suggested the possibility that viral sialidase activity plays a new significant role at the early stage of the IAV lifecycle such as the virus entry process into cells. A 19-amino-acid deletion in the stalk region of IAV NA increased virus replication in chicken cells without affecting the release process of progeny virus at the late stage of the IAV lifecycle (18). Glucosyl hesperidin, a derivative of a flavonoid obtained from citrus fruits, suppresses IAV infection at the early stage, probably by the inhibitory effect of IAV sialidase activity, with no inhibitory effect on the virus receptor binding and with no cytotoxicity (61). Zanamivir inhibits IAV infection at the early stage by deep cellular membrane embedding of IAV with no effect on virus receptor binding (cell surface adsorption) or on hemolysis activity (fusion process between the cell membrane and viral membrane) (62). Treatment with oseltamivir carboxylate, one of the IAV and influenza B virus NA-specific inhibitors, at the early stage of cell surface adsorption and cell entry of IAV inhibited virus infection (63). Thus, sialidase activity of IAV is likely to play a significant role not only at the late stage but also at the early stage of the IAV lifecycle.

\section{E. Analysis of Pandemic Occurrence Based on Low- pH Stability of IAV Sialidase Activity \\ E-1. 1968 Pandemic IAV}

Since all pandemic IAVs have low-pH stable NA, it is expected that the low-pH-stable NA contributes to the occurrence of a pandemic of a new subtype of IAV. 1968 pandemic H3N2 IAV occurred by genetic introduction of the H3 HA gene from avian IAV to human epidemic H2N2 IAV originating from 1957 pandemic
H2N2 IAV (64). The viral RNA polymerase subunit PB1 gene of 1968 pandemic IAV is believed to have been inherited from that of avian IAV (65). Description in this review is limited to HA and NA. N2 NAs of human epidemic H2N2 IAV in 1968 were divided into two types, low-pH-stable NA and low-pH-unstable NA. These types can also be definitely divided by phylogenetic analysis of N2 NA genes $(48,66)$. The low-pH-stable NA of 1968 pandemic H3N2 IAV is derived from that of human epidemic H2N2 IAV. It is thought that acquisition of low-pH-stable NA contributes to infection spread of a new pandemic IAV probably by enhancement of virus replication. Human epidemic H3N2 IAV NAs after 1971 show no low-pH stability. The low-pH-unstable NA of human epidemic H3N2 IAV after 1971 is derived from that of human epidemic H2N2 IAV before 1968, which is a phylogenetically different lineage from the low-pH-stable NA of human epidemic H2N2 IAV (Fig. 4). From phylogenetic analysis, it is predicted that human epidemic H3N2 IAVs in 1969, A/Taiwan/1/1969 (H3N2) and A/England/878/1969 (H3N2), had lost the low-pH stability by introduction of the low-pH-unstable NA of human H2N2 IAV, although the sialidase activities were not measured (66). Genetic reassortment between the low-pH-stable NA of 1968 pandemic H3N2 IAV and the low-pH-unstable NA of human H2N2 IAV probably occurred by simultaneous infection of both IAVs in humans around 1968. It is thought that loss of the low-pH stability of NA is involved in adaptation of an epidemic of a new subtype of IAV after a pandemic, although the reason is not clear. In a pandemic, a large number of humans should acquire immunization against the pandemic IAV. This would result in a drastic decrease in the number of humans susceptible to the pandemic IAV. The number of susceptible (non-immunized) humans may remain rela-

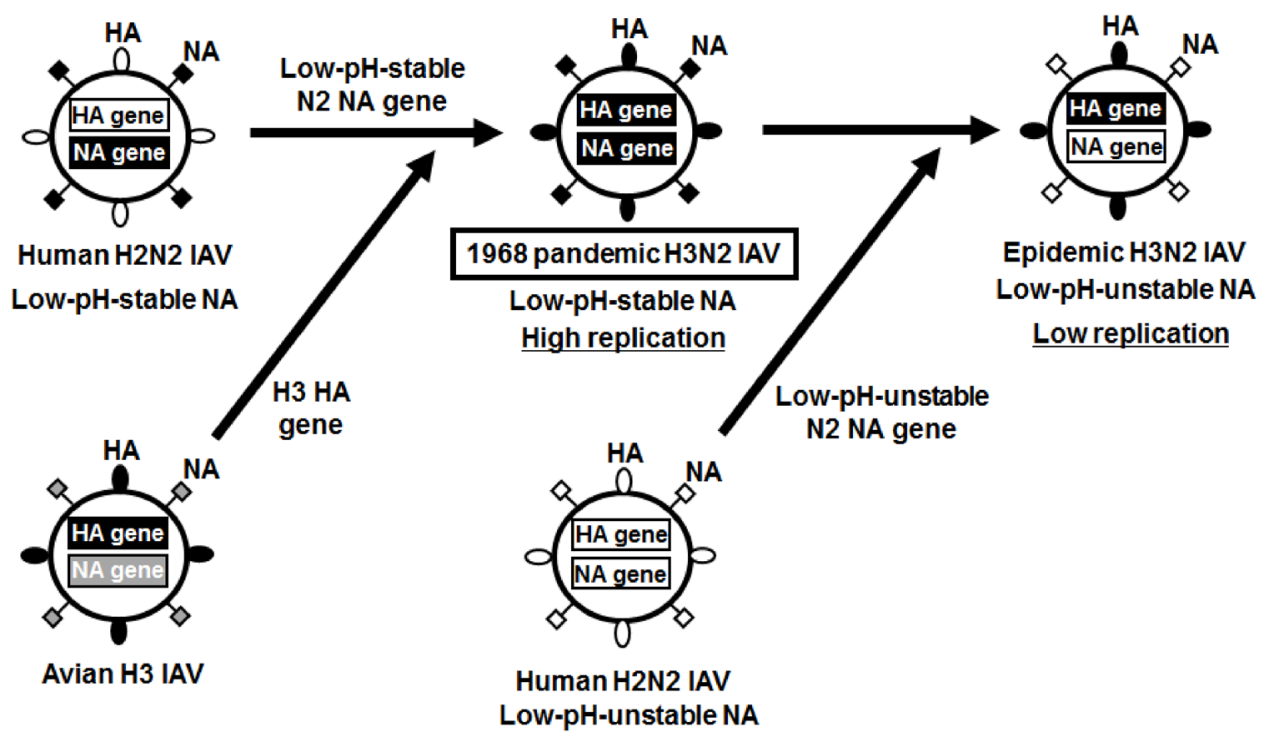

Fig. 4. Transition of the low-pH stability of IAV sialidase activity in the 1968 pandemic. 

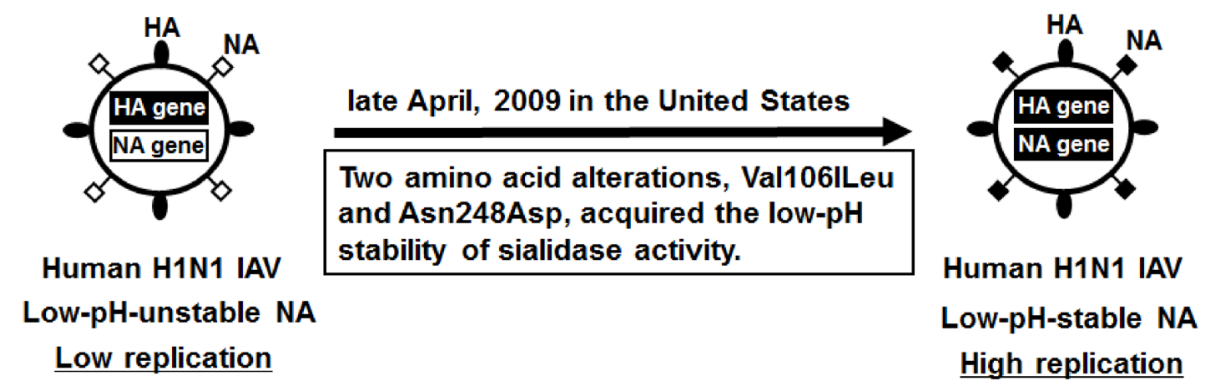

Fig. 5. Acquisition of the low-pH stability of IAV sialidase activity in the 2009 pandemic.

tively constant by a decrease of virus replication ability through the loss of low-pH stability of sialidase activity, leading to an epidemic. Once IAV becomes epidemic, epidemic IAV can escape from the acquired immune system by annual antigenic variation in viral surface antigens. An epidemic is thought to be stable state for annual continuous infections of IAV in humans. One way of the coexistence may be loss of the low-pH stability of sialidase activity.

\section{E-2. 2009 Pandemic IAV}

2009 pandemic H1N1 IAV occurred from direct transmission of swine IAV to humans. The NA gene of 2009 pandemic IAV is derived from that of the Eurasia-type swine IAV, which emerged from direct transmission of avian H1N1 IAV to pigs in 1979 (67). There were also two types of NA in 2009 pandemic IAV, low-pHstable NA and low-pH-unstable NA. For the low-pH-unstable NA of 2009 pandemic A/California/04/2009 (H1N1), two amino acid alterations of Val106Ile and Asn248Asp resulted in acquisition of low-pH stability. At the early stage of the 2009 pandemic in March and early April in 2009, the IAV mainly had low-pH-unstable NA with Val and Asn at positions 106 and 248, respectively. After late April (April 19 at the earliest) in 2009, low-pH-stable NA with Ile and Asp at positions 106 and 248, respectively, appeared and predominantly increased in the United States (Fig. 5) (41). In fact, for 394 patients with 2009 pandemic IAV infection in the United States from March 28 to May 5 in 2009, the number of patients drastically increased after April 19 in 2009 on date of illness onset (68). This seasonal coincidence between acquisition of low$\mathrm{pH}$-stable NA and increase of patients strongly suggests that the low-pH-stable NA contributed to the 2009 pandemic probably by enhancement of virus replication. It is expected that the low-pHstable NA of 2009 pandemic IAV will lose its low-pH stability together with adaptation to an epidemic, as appeared in a previous pandemic.

\section{F. Fluorescent Substrates for Detection of IAV Siali- dase Activity}

Some sialidase substrates are used as commercial reagents for detection of sialidase activity. For fluorescent detection of IAV sialidase activity, 4-methylumberlliferyl-Neu5Ac (4MU-Neu5Ac) has been most often used (69). Sialidase activity hydrolyzes 4MUNeu5Ac into Neu5Ac and the soluble fluorescent compound 4-methylumberlliferone. Although 4MU-Neu5Ac is highly sensitive for sialidase detection, it cannot histochemically show localization of sialidase activity. When 5-bromo-4-chloroindol-3-ylNeu5Ac (X-Neu5Ac) is incubated together with the sensitizer Fast Red Violet LB, the insoluble fluorescent compound produced can histochemically and fluorescently show localization of IAV sialidase activity (57). However, nonspecific staining other than staining of the IAV-infected cells is readily enhanced by Fast Red Violet LB. X-Neu5Ac also does not have sufficient sensitivity to sialidase activity. An insoluble fluorescent compound that can locally deposit on cells and tissues is needed for histochemical fluorescent visualization of sialidase activity. Recently, a highly sensitive sialidase substrate with an insoluble fluorescent compound named BTP3 has been developed as a new fluorescent sialidase detection tool for histochemical visualization of sialidase activity. BTP3, a benzothiazolylphenol derivative, is a crystalline, insoluble, acidresistant, and fluorescently stable compound (excitation/emission $=372 / 526 \mathrm{~nm})(70-73)$. BTP3-Neu5Ac was synthesized as a sialidase substrate by conjugation of Neu5Ac to BTP3. BTP3Neu5Ac itself is non-fluorescent. After cleavage of Neu5Ac from BTP3-Neu5Ac by sialidase reaction, fluorescent BTP3 is locally deposited at the location of sialidase activity (Fig. 6A).

Viruses showing sialidase activity include influenza viruses (IAV and influenza B virus) and some parainfluenza viruses. Reactivity to BTP3-Neu5Ac has been reported in sialidase activities of IAV, influenza B virus, Sendai virus (rodent pathogenic parainfluenza virus), and Newcastle disease virus (avian pathogenic parainfluenza virus). These viruses bear sialidase on the viral surface. Also, cells infected with these viruses abundantly express viral sialidase on the cell surface. BTP3-Neu5Ac can fluorescently visualize these viruses dot-blotted on the membrane and the infected cells through high activity of viral sialidase in a short time (within $10 \mathrm{~min}$ ) and with an easy protocol of BTP3-Neu5Ac addi- 
A
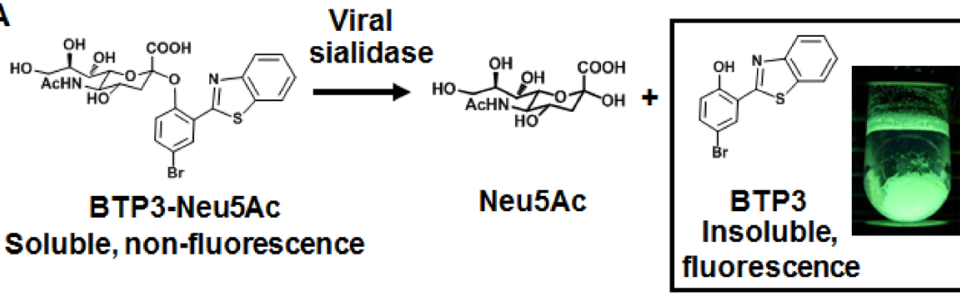

No fixation

No antibody

Easy protocol

Rapid detection

Soluble, non-fluorescence

fluorescence

Fluorescent

visualization of virus

and virus-infected cells
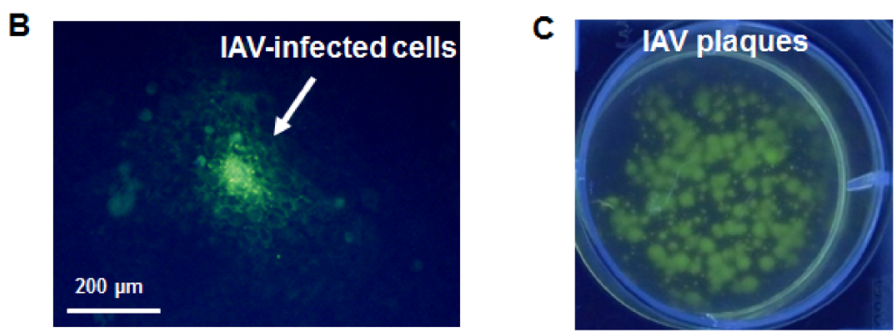

Fig. 6. Fluorescent visualization of IAV sialidase activity by BTP3-Neu5Ac. A, Scheme of BTP3-Neu5Ac reaction for fluorescent visualization of IAV sialidase activity. B, Fluorescent visualization of IAV-infected cells after $12 \mathrm{~h}$ of infection by BTP3-Neu5Ac. C, Fluorescent visualization of IAV plaques after 2 days of infection by BTP3-Neu5Ac.

tion only (Fig. 6B) (74-76). Immunostaining with a viral antigenspecific antibody has been the conventional method for staining infected cells. BTP3-Neu5Ac can stain infected cells without the need for specific antibodies against viruses of variable antigenicities. For various subtypes of IAV, it has been confirmed that BTP3Neu5Ac can detect infected cells or NA-expressing cells of human H1N1, human H3N2, 1918 pandemic H1N1, 2009 pandemic H1N1, HPAI H5N1, avian H5N3, and avian H7N9 (isolated from humans in 2013) IAVs, regardless of the IAV subtype and host. BTP3-Neu5Ac can stain living infected cells without the need for fixation of cells and tissues. Viral plaque (a population of infected cells) is formed by culture of infected cells on a agarosecontaining medium. Since the plaque becomes visible by omission of the population of dead infected cells, clear plaque formation is dependent on viral cytotoxicity. Some stains show no clear visible plaque because of low viral cytotoxicity. Viral plaques (plaque-like populations of infected cells) are fluorescently visualized by addition of BTP3-Neu5Ac on the agarose-containing medium, even for a virus strain showing no clear plaque (Fig. 6C). At that time, since the virus maintains infectivity because of no cell fixation, isolation of the virus strain can be performed by infection of fresh cultured cells with a fluorescent plaque. When mice are intranasally inoculated with IAV, BTP3-Neu5Ac can also stain the infected regions (likely to be the peribronchiolar epithelium) in a lung section fixed with paraformaldehyde, which retains $50-70 \%$ of viral sialidase activity before fixation (74). Since an optical $\mathrm{pH}$ region of Sendai virus sialidase is an acidic condition ( $\mathrm{pH} 4.0-5.0)$, acidic resistance of BTP3 fluorescence is suitable for its sialidase detection (76). BTP3-Neu5Ac would contribute to academic research and hygiene surveys as an efficient tool for visualization of infected regions, confirmation of virus culture, and virus isolation.

\section{G. Perspective}

Sialidase activity is an essential factor in efficient replication of IAV. Therefore, many influenza virus drugs, including commercial drugs and drugs under study, target inhibition of viral sialidase activity. IAV sialidase activity likely affects not only the well-known late stage (release of progeny virus) but also the early stage (receptor binding and cell entry) of the IAV life cycle. However, the function of sialidase activity at the early stage is unclear. Questions regarding IAV sialidase activity remain to be answered. Pandemic occurrence is thought to be mainly attributed to no immunization of humans against new antigenicities of HA and NA on the surface of the new subtype of IAV. Since genetic reassortment between IAVs can readily occur in an experiment, it is predicted that natural genetic reassortment of IAVs also occurs at a certain frequency. However, a historical pandemic emerges only every few decades. Factors other than change of IAV subtype also appear to be involved in natural pandemic occurrence. As one such factor, the low-pH stability of IAV sialidase activity probably contributes to efficient infection spread in a pandemic. By development of the new sialidase substrate BTP3-Neu5Ac, high sialidase activity can also be utilized as a landmark of the location of virus and infected cells. IAV sialidase activity is expected to have many applications for academic research and hygiene, such as a drug target, pandemic prediction, and confirmation of infected cells. Mammalian sialidase is involved in various biological functions and diseases such as cancer progression and axon regeneration in the peripheral 
nervous system $(77,78)$. Further study on IAV sialidase may contribute to an understanding of mammalian sialidase functions and sialidase-associated diseases.

\section{Acknowledgments}

I would like to acknowledge people in my laboratory who contributed to my work on viral sialidase. I would also like to acknowledge Dr. Takashi Suzuki (University of Shizuoka), Dr. Yasuo Suzuki (University of Shizuoka, Chubu University), Dr. Kennedy F. Shortridge (University of Hong Kong), Dr. Takehiko Saito (National Institute of Infectious Diseases, National Institute of Animal Health), Dr. Hideo Goto (University of Tokyo), Dr. Yoshihiro Kawaoka (University of Tokyo and University of WisconsinMadison), Dr. Jiasheng Song (University of Wisconsin-Madison), and Dr. Tadamune Otsubo and Dr. Kiyoshi Ikeda (Hiroshima International University). Finally, I would like to acknowledge financial support from University of Shizuoka, the Mochida Memorial Foundation for Medical and Pharmaceutical Research, the SRI
(Shizuoka Research Institute) academic research grant, Sasakawa Scientific Research Grant from the Japan Science Society (23439), Hokuto Foundation for Bioscience, Research Foundation for the Electrotechnology of Chubu, Nakatani Foundation for Advancement of Measuring Technologies in Biomedical Engineering, the Kurata Memorial Hitachi Science and Technology Foundation, the Futaba Electronics Memorial Foundation, Tokai Foundation for Technology, Takahashi Industrial and Economic Research Foundation, Izumi Science and Technology Foundation, The Shimabara Science Promotion Foundation, Japan Foundation for Aging and Health, and the MEXT/JSPS KAKENHI (Challenging Exploratory Research, 26670064; Young Scientists A, 15H05644) and in part from the MEXT/JSPS KAKENHI (Scientific Research B, 18390142; Scientific Research C, 23590549), Institution for Fermentation, Osaka, CREST (Japan Science and Technology Agency), and the Gobal COE Program from the Japan Society for the Promotion of Science.

\section{References}

1. Suzuki, Y. (2005) Biol. Pharm. Bull. 28, 399-4082.

2. Colman, P. M., Varghese, J. N., and Laver, W. G. (1983) Nature 303, 41-44.

3. Varghese, J. N., and Colman, P. M. (1991) J. Mol. Biol. 221, 473-486.

4. Palese, P., Tobita, K., Ueda, M., and Compans, R. W. (1974) Virology 61, 397-410.

5. von Itzstein, M., Wu, W. Y., Kok, G. B., Pegg, M. S., Dyason, J. C., Jin, B., Van Phan, T., Smythe, M. L., White, H. F., Oliver, S. W., Colman, P. M., Varghese, J. N., Ryan, D. M., Woods, J. M., Bethell, R. C., Hotham, V. J., Cameron, J. M., and Penn, C. R. (1993) Nature 363, $418-423$.

6. Li, Q., Sun, X., Li, Z., Liu, Y., Vavricka, C. J., Qi, J., and Gao, G. F. (2012) Proc. Natl. Acad. Sci. U.S.A. 109, $18897-18902$.

7. Zhu, X., Yang, H., Guo, Z., Yu, W., Carney, P. J., Li, Y., Chen, L. M., Paulson, J. C., Donis, R. O., Tong, S., Stevens, J., and Wilson, I. A. (2012) Proc. Natl. Acad. Sci. U.S.A. 109, 18903-18908.

8. Tong, S., Zhu, X., Li, Y., Shi, M., Zhang, J., Bourgeois, M., Yang, H., Chen, X., Recuenco, S., Gomez, J., Chen, L. M., Johnson, A., Tao, Y., Dreyfus, C., Yu, W., McBride, R., Carney, P. J., Gilbert, A. T., Chang, J., Guo, Z., Davis, C. T., Paulson, J. C., Stevens, J., Rupprecht, C. E., Holmes, E. C., Wilson, I. A., and Donis, R. O. (2013) PLoS Pathog. 9, e1003657.

9. Barman, S., and Nayak, D. P. (2000) J. Virol. 74, 6538-6545.

10. Barman, S., Adhikary, L., Chakrabarti, A. K., Bernas, C., Kawaoka, Y., and Nayak, D. P. (2004) J. Virol. 78, 5258-5269.

11. Zhou, H., Yu, Z., Hu, Y., Tu, J., Zou, W., Peng, Y., Zhu, J., Li, Y., Zhang, A., Yu, Z., Ye, Z., Chen, H., and Jin, M. (2009) PLoS ONE 17, e6277.

12. Wu, J., Zhang, F., Wang, M., Xu, C., Song, J., Zhou, J., Lin, X., Zhang, Y., Wu, X., Tan, W., Lu, J., Zhao, H., Gao, J., Zhao, P., Lu, J., and Wang, Y. (2010) PLoS ONE 5, e15825.

13. Matsuoka, Y., Swayne, D. E., Thomas, C., Rameix-Welti, M. A., Naffakh, N., Warnes, C., Altholtz, M., Donis, R., and Subbarao, K. (2009) J. Virol. 83, 4704-4708.

14. Saito, T., Taylor, G., Laver, W. G., Kawaoka, Y., and Webster, R. G. (1994) J. Virol. 68, 1790-1796.

15. Hausmann, J., Kretzschmar, E., Garten, W., and Klenk, H. D. (1997) J. Gen. Virol. 78, 3233-3245.

16. Colman, P. M., Laver, W. G., Varghese, J. N., Baker, A. T., Tulloch, P. A., Air, G. M., and Webster, R. G. (1987) Nature 326, $358-363$.

17. Kobasa, D., Wells, K., and Kawaoka, Y. (2001) J. Virol. 75, 11773-11780.

18. Munier, S., Larcher, T., Cormier-Aline, F., Soubieux, D., Su, B., Guigand, L., Labrosse, B., Cherel, Y., Quéré, P., Marc, D., and Naffakh, N. (2010) J. Virol. 84, 940-952.

19. Li, S., Schulman, J., Itamura, S., and Palese, P. (1993) J. Virol. 67, 6667-6673.

20. Burmeister, W. P., Cusack, S., and Ruigrok, R. W. (1994) J. Gen. Virol. 75, 381-388.

21. Dimmock, N. J. (1971) J. Gen. Virol. 13, 481-483.

22. Brett, I. C., and Johansson, B. E. (2006) J. Biochem. 139, 439-447.

23. Chong, A. K., Pegg, M. S., and von Itzstein, M. (1991) Biochim. Biophys. Acta 1077, 65-71.

24. Johansson, B. E., and Brett, I. C. (2003) J. Biochem. 134, 345-352.

25. Baum, L. G., and Paulson, J. C. (1991) Virology 180, 10-15.

26. Kobasa, D., Kodihalli, S., Luo, M., Castrucci, M. R., Donatelli, I., Suzuki, Y., Suzuki, T., and Kawaoka, Y. (1999) J. Virol. 73, 6743-6751.

27. Uhlendorff, J., Matrosovich, T., Klenk, H. D., and Matrosovich, M. (2009) Arch. Virol. 154, 945-957. 
28. França de Barros, J. Jr., Sales Alviano, D., da Silva, M. H., Dutra Wigg, M., Sales Alviano, C., Schauer, R., and dos Santos Silva Couceiro, J. N. (2003) Intervirology 46, 199-206.

29. Sato, K., Hanagata, G., Kiso, M., Hasegawa, A., and Suzuki, Y. (1998) Glycobiology 8, 527-532.

30. Mochalova, L., Kurova, V., Shtyrya, Y., Korchagina, E., Gambaryan, A., Belyanchikov, I., and Bovin, N. (2007) Arch. Virol. 152, $2047-2057$.

31. Masuda, H., Suzuki, T., Sugiyama, Y., Horiike, G., Murakami, K., Miyamoto, D., Jwa Hidari, K. I., Ito, T., Kida, H., Kiso, M., Fukunaga, K., Ohuchi, M., Toyoda, T., Ishihama, A., Kawaoka, Y., and Suzuki, Y. (1999) FEBS Lett. 464, 71-74.

32. Takahashi, T., Hashimoto, A., Maruyama, M., Ishida, H., Kiso, M., Kawaoka, Y., Suzuki, Y., and Suzuki, T. (2009) FEBS Lett. 583, $3171-3174$.

33. Suzuki, T., Horiike, G., Yamazaki, Y., Kawabe, K., Masuda, H., Miyamoto, D., Matsuda, M., Nishimura, S. I., Yamagata, T., Ito, T., Kida, H., Kawaoka, Y., and Suzuki, Y. (1997) FEBS Lett. 404, 192-196.

34. Ito, T., Suzuki, Y., Suzuki, T., Takada, A., Horimoto, T., Wells, K., Kida, H., Otsuki, K., Kiso, M., Ishida, H., and Kawaoka, Y. (2000) J. Virol. 74, 9300-9305.

35. Suzuki, Y., Ito, T., Suzuki, T., Holland, R. E. Jr., Chambers, T. M., Kiso, M., Ishida, H., and Kawaoka, Y. (2000) J. Virol. 74, $11825-11831$.

36. Bateman, A. C., Karamanska, R., Busch, M. G., Dell, A., Olsen, C. W., and Haslam, S. M. (2010) J. Biol. Chem. 285, $34016-34026$.

37. Takahashi, T., Takano, M., Kurebayashi, Y., Masuda, M., Kawagishi, S., Takaguchi, M., Yamanaka, T., Minami, A., Otsubo, T., Ikeda, K., and Suzuki, T. (2014) J. Virol. 88, 8445-8456.

38. Stepushkin, V. A., Bukrinskaya, A. G., Prokazova, N. V., Zhigis, L. S., Reshetov, P. D., Shaposhnikova, J. I., and Bergelson, L. D. (1985) FEBS Lett. 182, 273-277.

39. Takahashi, T., Suzuki, Y., Nishinaka, D., Kawase, N., Kobayashi, Y., Hidari, K. I., Miyamoto, D., Guo, C. T., Shortridge, K. F., and Suzuki, T. (2001) J. Biochem. 130, 279-283.

40. Takahashi, T., Kurebayashi, Y., Ikeya, K., Mizuno, T., Fukushima, K., Kawamoto, H., Kawaoka, Y., Suzuki, Y., and Suzuki, T. (2010) PLoS ONE 5, e15556.

41. Takahashi, T., Song, J., Suzuki, T., and Kawaoka, Y. (2013) PLoS ONE 8, e64439.

42. Reid, A. H., Fanning, T. G., Janczewski, T. A., and Taubenberger, J. K. (2000) Proc. Natl. Acad. Sci. U.S.A. 97, 6785-6790.

43. Takahashi, T., Nidom, C. A., Quynh Le, M. T., Suzuki, T., and Kawaoka, Y. (2012) FEBS Open Bio 2, 261-266.

44. Oxford, J. S. (2000) Rev. Med. Virol. 10, 119-133.

45. Kilbourne, E. D. (2006) Emerg. Infect. Dis. 12, 9-14.

46. Webster, R. G., Yakhno, M., Hinshaw, V. S., Bean, W. J., and Murti, K. G. (1978) Virology 84, 268-278.

47. Kida, H., Yanagawa, R., and Matsuoka, Y. (1980) Infect. Immun. 30, 547-553.

48. Suzuki, T., Takahashi, T., Saito, T., Guo, C. T., Hidari, K. I., Miyamoto, D., and Suzuki, Y. (2004) FEBS Lett. 557, $228-232$.

49. Xu, X., Zhu, X., Dwek, R. A., Stevens, J., and Wilson, I. A. (2008) J. Virol. 82, 10493-10501.

50. Takahashi, T., Suzuki, T., Hidari, K. I., Miyamoto, D., and Suzuki, Y. (2003) FEBS Lett. 543, 71-75.

51. Baker, N. J., and Gandhi, S. S. (1976) Arch. Virol. 52, 7-18.

52. Varghese, J. N., Laver, W. G., and Colman, P. M. (1983) Nature 303, 35-40.

53. Varghese, J. N., Webster, R. G., Laver, W. G., and Colman, P. M. (1988) J. Mol. Biol. 200, 201-203.

54. Varghese, J. N., McKimm-Breschkin, J. L., Caldwell, J. B., Kortt, A. A., and Colman, P. M. (1992) Proteins 14, 327-332.

55. Li, Q., Qi, J., Zhang, W., Vavricka, C. J., Shi, Y., Wei, J., Feng, E., Shen, J., Chen, J., Liu, D., He, J., Yan, J., Liu, H., Jiang, H., Teng, M., Li, X., and Gao, G. F. (2010) Nat. Struct. Mol. Biol. 17, 1266-1268.

56. Russell, R. J., Haire, L. F., Stevens, D. J., Collins, P. J., Lin, Y. P., Blackburn, G. M., Hay, A. J., Gamblin, S. J., and Skehel, J. J. (2006) Nature 443, $45-49$.

57. Suzuki, T., Takahashi, T., Guo, C. T., Hidari, K. I., Miyamoto, D., Goto, H., Kawaoka, Y., and Suzuki, Y. (2005) J. Virol. 79, $11705-11715$.

58. Overly, C. C., Lee, K. D., Berthiaume, E., and Hollenbeck, P. J. (1995) Proc. Natl. Acad. Sci. U.S.A. 92, 3156-3160.

59. Patel, S., Ramakrishnan, L., Rahman, T., Hamdoun, A., Marchant, J. S., Taylor, C. W., and Brailoiu, E. (2011) Cell Calcium 50, $157-167$.

60. Sandhoff, K., and Harzer, K. (2013) J. Neurosci. 33, 10195-10208.

61. Saha, R. K., Takahashi, T., and Suzuki, T. (2009) Biol. Pharm. Bull. 32, 1188-1192.

62. Ohuchi, M., Asaoka, N., Sakai, T., and Ohuchi, R. (2006) Microbes Infect. 8, 1287-1293.

63. Matrosovich, M. N., Matrosovich, T. Y., Gray, T., Roberts, N. A., and Klenk, H. (2004) J. Virol. 78, 12665-12667.

64. Nakao, H., Nakajima, K., and Nakajima, S. (1993) J. Gen. Virol. 74, 1667-1672.

65. Kawaoka, Y., Krauss, S., and Webster, R. G. (1989) J. Virol. 63, 4603-4608.

66. Lindstrom, S. E., Cox, N. J., and Klimov, A. (2004) Virology 328, 101-119.

67. Neumann, G., Noda, T., and Kawaoka, Y. (2009) Nature 459, 931-939.

68. Dawood, F. S., Jain, S., Finelli, L., Shaw, M. W., Lindstrom, S., Garten, R. J., Gubareva, L. V., Xu, X., Bridges, C. B., and Uyeki, T. M.; Novel Swine-Origin Influenza A (H1N1) Virus Investigation Team. (2009) N. Engl. J. Med. 360, 2605-2615.

69. Nayak, D. P., and Reichl, U. (2004) J. Virol. Methods 122, 9-15.

70. Kim, T. I., Kang, H. J., Han, G., Chung, S. J., and Kim, Y. (2009) Chem. Commun. (Camb.) 39, 5895-5897.

71. Otsubo, T., Minami, A., Fujii, H., Taguchi, R., Takahashi, T., Suzuki, T., Teraoka, F., and Ikeda, K. (2013) Bioorg. Med. Chem. Lett. 23, 22452249.

72. Minami, A., Otsubo, T., Ieno, D., Ikeda, K., Kanazawa, H., Shimizu, K., Ohata, K., Yokochi, T., Horii, Y., Fukumoto, H., Taguchi, R., Takahashi, T., Oku, N., and Suzuki, T. (2014) PLoS ONE 9, e81941.

73. Takahashi, T., Otsubo, T., Ikeda, K., Minami, A., and Suzuki, T. (2014) Biol. Pharm. Bull. 37, 1668-1673.

74. Kurebayashi, Y., Takahashi, T., Otsubo, T., Ikeda, K., Takahashi, S., Takano, M., Agarikuchi, T., Sato, T., Matsuda, Y., Minami, A., Kanazawa, H., Uchida, Y., Saito, T., Kawaoka, Y., Yamada, T., Kawamori, F., Thomson, R., von Itzstein, M., and Suzuki, T. (2014) Sci. Rep. 4, 4877.

75. Takahashi, T., Takano, M., Agarikuchi, T., Kurebayashi, Y., Minami, A., Otsubo, T., Ikeda, K., and Suzuki, T. (2014) J. Virol. Methods 209, 136- 
142.

76. Takano, M., Takahashi, T., Agarikuchi, T., Kurebayashi, Y., Minami, A., Otsubo, T., Ikeda, K., Kanazawa, H., and Suzuki, T. (2014) Virology 464-465, 206-212.

77. Miyagi, T., Takahashi, K., Hata, K., Shiozaki, K., and Yamaguchi, K. (2012) Glycoconj. J. 29, 567-577.

78. Kappagantula, S., Andrews, M. R., Cheah, M., Abad-Rodriguez, J., Dotti, C. G., and Fawcett, J. W. (2014) J. Neurosci. 34, $2477-2492$.

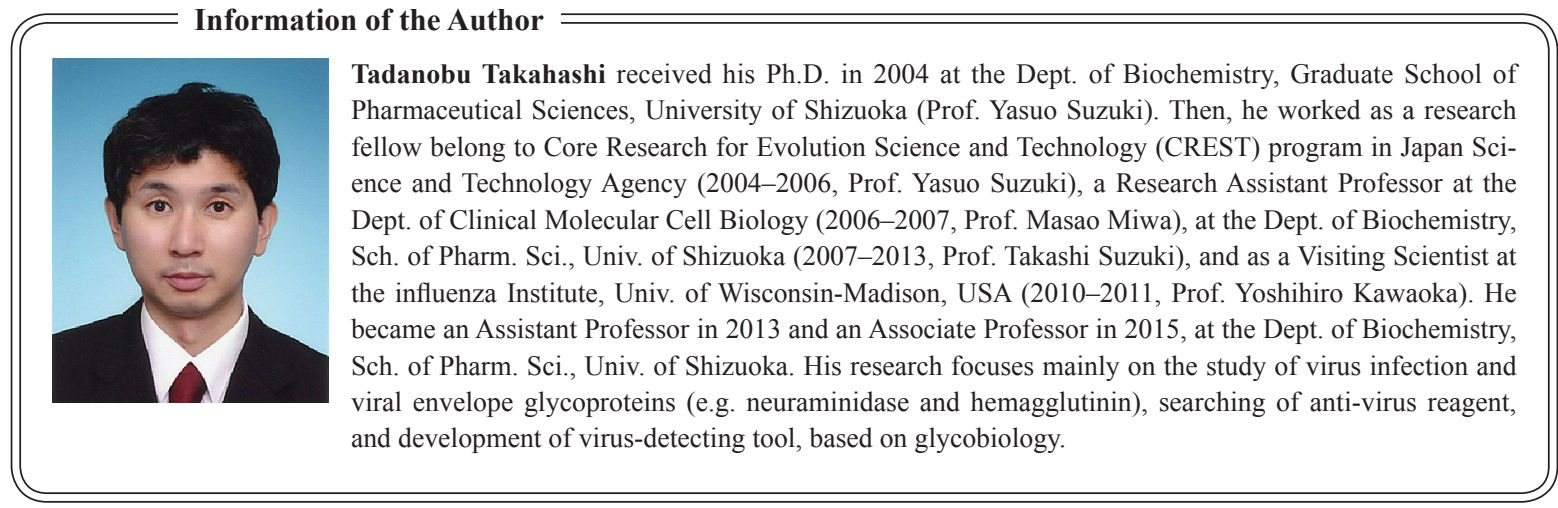

